\title{
Paraneoplastische limbische Enzephalitis bei kleinzelligem Bronchialkarzinom
}

\section{B. Fischer ${ }^{1}$ \\ P. Kalden ${ }^{2}$ \\ P. P. Urban ${ }^{3}$ \\ R. Buhl'}

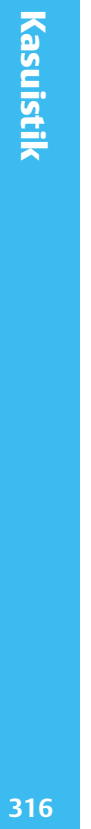

Paraneoplastic Limbic Encephalitis in Small Cell Lung Carcinoma

\section{Zusammenfassung}

Ein 63-jähriger Patient litt seit einigen Tagen unter Verwirrtheitszuständen. 3 Tage vor Krankenhauseinweisung hatte der Hausarzt im Röntgenbild der Thoraxorgane eine mediastinale Raumforderung festgestellt. Am Aufnahmetag in die Klinik erfolgte eine Computertomographie des Schädels, die überraschenderweise keinen Hinweis auf zerebrale Filiae ergab. Die Magnetresonanztomographie zeigte eine Signalhyperintensität im mesialen Temporallappen beidseits. Durch Mediastinoskopie wurde histologisch die Diagnose eines kleinzelligen Bronchialkarzinoms (SCLC) gesichert. Fernmetastasen wurden nicht festgestellt. Im Liquor cerebrospinalis und im Serum fanden sich antineuronale Antikörper (anti-Hu). In Zusammenschau des klinischen Bildes, des MRT-Befundes und des Nachweises antineuronaler Autoantikörper wurde die Diagnose einer paraneoplastischen limbischen Enzephalitis bei SCLC gestellt. Umgehend wurde eine Chemotherapie plus Immunsuppression durch Kortikoide eingeleitet. Während der Tumor auf die Chemotherapie ein gutes Ansprechen zeigte, war der Verlauf bezüglich der zentralnervösen Symptome unbefriedigend. Zwar besserten sich Halluzinationen und Agitiertheit, aber die erheblichen mnestischen Störungen und die Persönlichkeitsveränderung persistierten.

\section{Abstract}

We report about a 63-year-old male patient who complained of a recent onset of confusion. A mediastinal mass had been detected in his chest X-ray three days before admission to our clinic. Surprisingly, a CT scan of the brain revealed no signs of cerebral masses or oedema. MRI demonstrated bright hyperintense signals in the medial aspect of both temporal lobes. Biopsy by mediastinoscopy showed small cell lung carcinoma (SCLC). No distant metastases were found by staging procedures. Cerebrospinal fluid and serum contained antineuronal antibodies (anti-Hu). The combination of symptoms, MRI findings and paraneoplastic antibodies established the diagnosis of paraneoplastic limbic encephalitis in a patient with SCLC. Chemotherapy combined with immunosuppression by corticosteroids was started immediately. The primary tumour responded to therapy but improvement of cerebral symptoms was unsatisfactory. Severe memory loss and personality changes remained unchanged while there was a slight improvement in confusion and hallucinations.

Institutsangaben

${ }^{1}$ III. Medizinische Klinik und Poliklinik, Hämatologie-Onkologie-Pneumologie

(Direktor: Prof. Dr. Ch. Huber), Klinikum der Johannes Gutenberg-Universität, Mainz

${ }^{2}$ Radiologische Klinik und Poliklinik (Direktor: Prof. Dr. M. Thelen),

Klinikum der Johannes Gutenberg-Universität, Mainz

${ }^{3}$ Neurologische Klinik und Poliklinik (Direktorin: Prof. Dr. M. Dieterich),

Klinikum der Johannes Gutenberg-Universität, Mainz

Korrespondenzadresse

Dr. med. Berthold Fischer · III. Medizinische Klinik und Poliklinik, Hämatologie-Onkologie-

Pneumologie · Klinikum der Johannes Gutenberg-Universität · 55131 Mainz

E-mail: b.fischer@3-med.klinik.uni-mainz.de

Eingang: 26. Januar 2004 · Akzeptiert: 9. Februar 2004

Bibliografie

Pneumologie 2004; 58: 316-319 • @ Georg Thieme Verlag KG Stuttgart · New York

DOI $10.1055 / \mathrm{s}-2004-818380$

ISSN 0934-8387 
Ein 63-jähriger Patient wird wegen einer mediastinalen Raumforderung unbekannter Dignität sowie akut aufgetretener Desorientiertheit und Persönlichkeitsveränderung in die Klinik eingewiesen. Der Patient ist Raucher (70 pack-years). Anamnestisch sind ein teilthrombosiertes infrarenales Bauchaortenaneurysma, eine Psoriasis vulgaris und eine medikamentös eingestellte arterielle Hypertonie bekannt. An subjektiven Beschwerden gibt der Patient eine geringe Belastungsdyspnoe, Abgeschlagenheit seit einem „grippalen Infekt“ vor 3 Wochen und Bewusstseinsstörungen an. Gewichtsverlust von 3,5 kg in den vergangenen $3 \mathrm{Wo}$ chen. Kein Fieber, keine Schmerzen.

\section{Befund}

$180 \mathrm{~cm}$ großer, $95 \mathrm{~kg}$ schwerer Patient in gutem Allgemeinzustand und adipösem Ernährungszustand. Keine Trommelschlegelfinger. Unauffälliger internistischer Untersuchungsbefund. Der neurologische Untersuchungsbefund ist bis auf ausgeprägte Störungen des Kurzzeitgedächtnisses, psychomotorische Unruhe und räumliche und zeitliche Desorientiertheit unauffällig. $\mathrm{Zu}$ Hause habe der Patient fremdanamnestisch auch einen zerebralen Krampfanfall erlitten. Das psychiatrische Konsil ergibt ein delirantes Syndrom zunächst unklarer Ätiologie.

\section{Zusatzuntersuchungen}

Eine bereits ambulant durchgeführte thorakale Computertomographie (CT) zeigt die auf der Thoraxübersicht erkennbare, weichteildichte Raumforderung im vorderen oberen Mediastinum, nach rechts ausladend, in einer Größe von 4,6 ×5,4 cm und eine $2 \mathrm{~cm}$ durchmessende Raumforderung kranial des Oberlappenbronchus rechts (Abb.1).

Unter der Verdachtsdiagnose eines zerebral metastasierten Bronchialkarzinoms wird am Aufnahmetag eine Schädel-CT durchgeführt, die unerwartet keinen richtungweisenden pathologischen Befund aufweist, insbesondere keinen Nachweis von zerebralen Filiae.

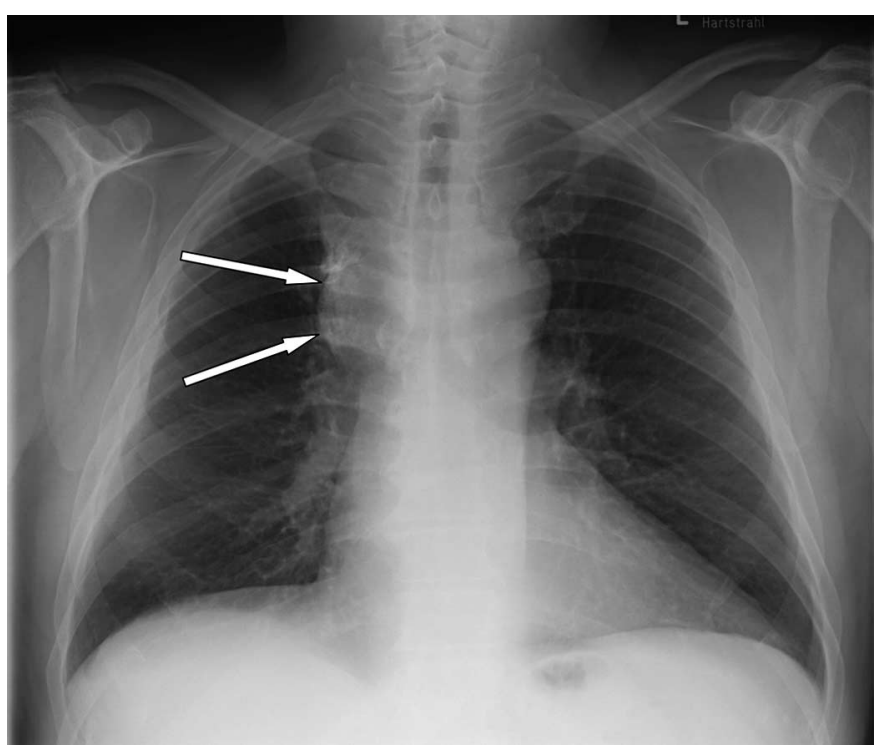

Abb. 1 Röntgen Thorax p.a. bei Aufnahme. Raumforderung rechts mediastinal (Pfeile).

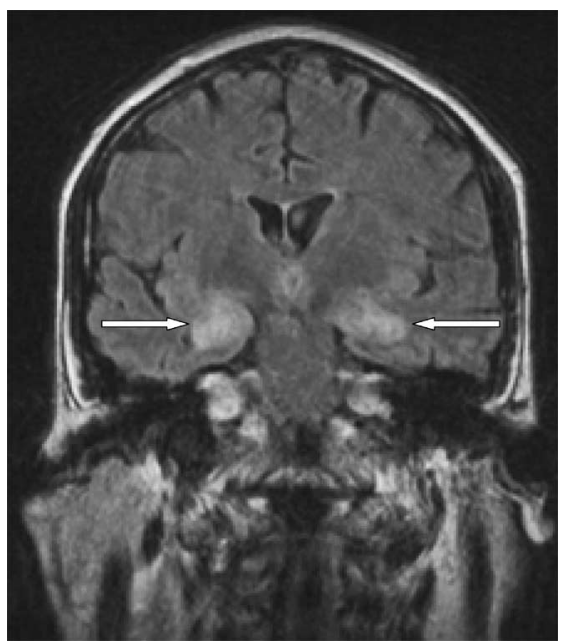

Abb. 2 MRT Schädel. $T_{2}$-gewichtete, liquorsupprimierte Sequenz in koronarer Schnittführung: Signalhyperintensität im mesialen Temporallappen beidseits (Pfeile).

Die mediastinoskopische Probeexzision der thorakalen Raumforderung ergibt Formationen eines kleinzelligen Bronchialkarzinoms (SCLC) mit starker Expression von TTF 1 und Synaptophysin sowie moderater Expression von Chromogranin. Die Reaktion für Zytokeratin 5/6 ist negativ.

Die Tumorausbreitungsdiagnostik inklusive Knochenszintigramm und Abdomen-CT verläuft ohne Anhalt für Fernmetastasen. Die Magnetresonanztomographie des Schädels (MRT) zeigt dann in der $\mathrm{T}_{2}$-gewichteten, liquorsupprimierten Sequenz eine Signalhyperintensität im mesialen Temporallappen beidseits vereinbar mit einer limbischen Enzephalitis (Abb. 2).

Laborchemisch auffällig ist eine Hyponatriämie $(128 \mathrm{mmol} / \mathrm{l}$ [Soll: 135-144]) im Sinne eines Syndroms der inadäquaten ADH-Sekretion und die pathologisch erhöhte neuronenspezifische Enolase von 30,5 ng/ml (Norm <17,5). Cyfra $21-1$ ist mit $1,28 \mathrm{ng} / \mathrm{ml}$ (Norm <3,3) unauffällig. Der Liquor zeigt lediglich eine geringe Erhöhung des Eiweißes auf $85,3 \mathrm{mg} / \mathrm{dl}($ Norm < 50) bei normaler Zellzahl. Nachdem die MRT den Befund einer limbischen Enzephalitis erbracht hatte, wird auf neuronale Autoantikörper im Liquor und Serum untersucht. Sowohl im Liquor als auch im Serum sind anti-Hu-Antikörper stark positiv (Tab.1).

\section{Diagnose}

Paraneoplastische limbische Enzephalitis bei kleinzelligem Bronchialkarzinom, limited disease.

\section{Therapie und Verlauf}

Der Patient muss wegen zunehmender deliranter Symptome mit passageren Halluzinationen und paranoiden Denkstörungen vorübergehend mit Haloperidol (z. B.: Haldol-Janssen ${ }^{\mathrm{R}}$ ) und Melperon (z.B. Eunerpan ${ }^{R}$ ) behandelt werden. Eine Behandlungspflegschaft durch die Ehefrau wird eingerichtet.

Umgehend wird eine zytostatische Chemotherapie bestehend aus Cisplatin/Etoposid eingeleitet. Vier dreiwöchige Kurse Etoposid $100 \mathrm{mg} / \mathrm{m}^{2}$ Tag 1, 2 und 3 plus Cisplatin $80 \mathrm{mg} / \mathrm{m}^{2}$ Tag 1 werden durchgeführt. Zeitgleich wird zur Behandlung der limbi- 
Tab. 1 Autoantikörpernachweis

\begin{tabular}{lll}
\hline Bestimmung & Ergebnis & Referenz \\
\hline $\begin{array}{l}\text { Liquor: } \\
\text { Anti-Hu-Antikörper } \\
\text { (Neuronenkerne + periphere Neurone) }\end{array}$ & positiv & negativ \\
\hline $\begin{array}{l}\text { Anti-Ri-Antikörper (Neuronenkerne) } \\
\text { Anti-Yo-Antikörper (Purkinje-Zellen) }\end{array}$ & negativ & negativ \\
\hline $\begin{array}{l}\text { Serum: } \\
\text { Anti-Hu-Antikörper } \\
\text { (Neuronenkerne + periphere Neurone) }\end{array}$ & negativ & negativ \\
\hline $\begin{array}{l}\text { Anti-Ri-Antikörper (Neuronenkerne) } \\
\text { Anti-Yo-Antikörper (Purkinje-Zellen) }\end{array}$ & negativ & \\
\hline
\end{tabular}

schen Enzephalitis eine Immunsuppression mit Prednisolon $100 \mathrm{mg}$ täglich begonnen.

Bereits nach dem ersten Chemotherapiezyklus ist eine Rückbildung der mediastinalen Raumforderung erkennbar. Der Patient muss allerdings zwischen Kurs 1 und 2 der Chemotherapie bei zunächst noch zunehmender Verwirrtheit in der Psychiatrischen Klinik stationär behandelt werden. Nach Gabe des zweiten Chemotherapiezyklus kann der Patient zwischen den Chemotherapiekursen bei leichter Besserung der Verwirrtheit und der Gedächtnisstörungen unter der Auflage der häuslichen Betreuung durch die Angehörigen entlassen werden. Nach insgesamt vier zytostatischen Zyklen ist eine partielle Remission eingetreten.

Der Patient fühlt sich subjektiv deutlich gebessert. Weiterhin bestehen allerdings deutliche Störungen des Kurzzeitgedächtnisses.

Eine konsolidierende mediastinale Radiatio wird angeschlossen. Diese muss allerdings wegen wieder zunehmender Verwirrtheit und Verschlechterung des Allgemeinzustandes nach insgesamt 25 Gy Herddosis abgebrochen werden. Zu diesem Zeitpunkt befindet sich das kleinzellige Bronchialkarzinom weiterhin in partieller Remission.

\section{Diskussion}

Die limbische Enzephalitis gehört zu den paraneoplastischen Syndromen (PNS).

PNS sind Begleitreaktionen maligner Tumoren, die durch vom Tumor freigesetzte hormonähnliche Substanzen oder immunologische Mechanismen hervorgerufen werden.

PNS können in vier Hauptgruppen eingeteilt werden:

- endokrine PNS (z. B. Syndrom der inadäquaten ADH-Sekretion [SIADH]),

- neurologische/neuromuskuläre PNS (z. B. limbische Enzephalitis; z. B. myasthenisches Syndrom [Lambert-Eaton]),

- hämatologische PNS (z. B. Thrombozytose) und

- dermatologische PNS (z. B. Acanthosis nigricans).
Die Prävalenz der PNS liegt beim Bronchialkarzinom zwischen 10 und $20 \%$ [1].

Die limbische Enzephalitis ist eine seltene neurologische Paraneoplasie, die sich durch schwere Störungen des Kurzzeitgedächtnisses (subakut, im Verlauf einiger Wochen), Persönlichkeitsveränderungen, weitere psychiatrische Symptome (z.B. Halluzinationen, Agitiertheit, Konfusion) und epileptische Anfälle äußert [2].

In nahezu allen Fällen liegt ein Tumorleiden vor bzw. wird im weiteren kurzfristigen Verlauf evident. Dabei liegt am häufigsten ein kleinzelliges Bronchialkarzinom vor. Weitere Tumorentitäten, die von einer limbischen Enzephalitis kompliziert werden können sind: nicht-kleinzelliges Bronchialkarzinom, Keimzelltumoren des Hodens, Mammakarzinom, Hodgkin-Lymphom, Teratome und malignes Thymom.

Sehr selten tritt eine limbische Enzephalitis ohne Tumorerkrankung auf. Es wird angenommen, dass es sich bei diesen seltenen Fällen um okkulte Tumorerkrankungen handelt [3].

Die neurologischen Symptome treten wie im vorgestellten Fall meistens (60\% der Patienten) vor Diagnose der Tumorerkrankung auf (im Mittel 3-4 Monate) und es handelt sich überwiegend um noch limitierte, nicht metastasierte Tumorerkrankungen mit der Option zur kurativ intendierten Therapie.

Histologisch finden sich bei der limbischen Enzephalitis perivaskuläre und parenchymale, lymphozytenreiche, entzündliche Infiltrationen durch $\mathrm{CD}^{+}-$-T-Zellen mit Neuronenuntergang und Gliose. Die Ursache der Entzündung ist bisher nicht endgültig aufgeklärt. Es wird angenommen, dass ein Autoimmunphänomen gegen Tumorantigene vorliegt, die eine Kreuzreaktivität mit Antigenen neuronaler Zellen aufweisen. Diese Hypothese wird unterstützt durch den Nachweis der anti-Hu-Autoantikörper bei $60 \%$ der Patienten [2].

Der anti-Hu-Autoantikörper mit einem Molekulargewicht von 35-40 kD erkennt als Antigen eine Familie RNA-prozessierender Proteine, die auf allen SCLC und auf neuronalen Zellen exprimiert werden [4]. Niedrige Titer des anti-Hu-Antikörpers werden auch ohne klinisches Beschwerdebild angetroffen [5]. Als signifikanter Titer von anti-Hu im Serum, der mit klinischen Beschwerden im Sinne einer paraneoplastischen Enzephalomyelitis einhergeht, gilt $1: 1000$. Der hier vorgestellte Patient wies anti-Hu-Antikörper in einer Titerstufe von 1:1600 auf.

In einer Serie von 196 Patienten ohne klinische Zeichen einer paraneoplastischen Enzephalomyelitis mit SCLC konnte die spanische Studiengruppe um Rosell bei 16\% einen niedrigen Titer von anti-Hu-Antikörpern nachweisen. Der Nachweis der anti-HuAntikörper war ein unabhängiger positiver Prognosefaktor für das Erreichen einer kompletten Remission und für längeres Überleben [6].

Die führenden neurologischen Auffälligkeiten bei Vorliegen einer paraneoplastischen Enzephalomyelitis und Nachweis von anti-Hu-Antikörpern aus einer Serie von 200 Patienten aus der Literatur gibt Tab. 2 wieder [7]. 
Tab. 2 Führendes neurologisches Beschwerdebild bei 200 Patienten (74\% kleinzelliges Bronchialkarzinom) mit anti-Huassoziierter Enzephalomyelitis [7]

\begin{tabular}{|ll}
\hline sensorische Neuropathie & $54 \%$ \\
\hline zerebelläre Symptome & $11 \%$ \\
\hline limbische Enzephalitis & $10 \%$ \\
\hline Hirnstammsymptome & $6 \%$ \\
\hline sensomotorische Neuropathie & $4 \%$ \\
\hline Dysautonomie, z.B. Pseudoobstruktion & $4 \%$ \\
\hline multifokale Symptome & $11 \%$ \\
\hline
\end{tabular}

Weitere Autoantikörper, die bei Erkrankung an kleinzelligem Bronchialkarzinom mit neurologischen Paraneoplasien vorkommen, sind: anti-Yo (zerebelläre Degeneration), anti-Ri (Myoklonie), anti-VGCC (Lambert-Eaton myasthenisches Syndrom), antiretinale Antikörper (tumorassoziierte Retinopathie) und Antiamphiphysin („stiff-man“-Syndrom, limbische Enzephalitis) [8].

Die klinische Diagnose der limbischen Enzephalitis erfordert nach den Kriterien von Gultekin [2] folgende Befunde:

1. typische Klinik,

2. Zeitraum unterhalb von 4 Jahren zwischen Entwicklung der neurologischen Symptome und der Diagnose der Tumorerkrankung,

3. Ausschluss anderer erklärender neuropathologischer Befunde, insbesondere Filiae,

4. eines der folgenden Befunde:

- entzündliche Liquorveränderung bei negativer Zytologie,

- Temporallappenveränderung im MRT Schädel,

- EEG: Epilepsieherd im Temporallappen.

Die CT des Schädels ergibt typischerweise keinen richtungweisenden Befund.

Die Signalveränderungen im MRT Schädel werden bei $57 \%$ der Patienten nachweisbar [2]. In der akuten Phase findet sich auch ein Anstieg in der Glukoseutilisation mit entsprechender Aktivitätssteigerung in der Positronenemissionstomographie.

Bioptische Verfahren sind von untergeordneter Bedeutung.

Der Nachweis der Autoantikörper gelingt in mehr als 50\% der Fälle, ist aber demzufolge keine Voraussetzung zur Diagnose.
Generell ist die therapeutische Beeinflussbarkeit der neurologischen Ausfälle, insbesondere der Gedächtnisstörungen, limitiert. Der größte Nutzen wird der Therapie der zugrundeliegenden Tumorerkrankung zugeschrieben. Immunsuppressive bzw. immunmodulatorische Behandlungen (z.B. Kortikosteroide bzw. Immunglobuline) werden häufig ergänzend eingesetzt. Dennoch wird nur bei einer Minderheit der Patienten eine Verbesserung der neurologischen Symptomatik erreicht [7]. Nur in seltenen Fällen kommt es zur weitgehenden Rückbildung der Enzephalopathie durch Remission der verantwortlichen Neoplasie.

Zusammenfassend sei durch diese Fallbeschreibung auf die Differenzialdiagnose der paraneoplastischen limbischen Enzephalitis bei Patienten mit Gedächtnisstörung, psychiatrischen Auffälligkeiten und zerebralen Anfällen hingewiesen, insbesondere bei Vorliegen eines kleinzelligen Bronchialkarzinoms und unauffälliger Schädel-CT. Die Diagnose wird durch das Schädel-MRT gestellt. Der Nachweis von Autoantikörpern ist ein weiterer diagnostischer Hinweis, ist aber nicht obligat. Die möglichst frühe Diagnose und Therapie der zugrundeliegenden Tumorerkrankung stellt auch die wirksamste Behandlungsoption des neurologischen Beschwerdebildes dar.

\section{Literatur}

${ }^{1}$ Thomas M, Gatzemeier U, Goerg R et al. Empfehlungen zur Diagnostik des Bronchialkarzinoms. Pneumologie 2000; 54: 361 - 371

${ }^{2}$ Gultekin SH, Rosenfeld MR, Voltz R et al. Paraneoplastic limbic encephalitis: neurological symptoms, immunological findings and tumour association in 50 patients. Brain 2000; 123: $1481-1494$

${ }^{3}$ Ravin CE, Bergin D, Bisset GS et al. Image interpretation session: 2000; Case 4. RadioGraphics 2001; 21: 267-287

${ }^{4}$ Szabo A, Dalmau J, Manley G et al. HuD, a paraneoplastic encephalomyelitis antigen, contains $\mathrm{R}$ binding domains and is homologous to Elav and Sex-lethal. Cell 1991; 67: 325 - 333

${ }^{5}$ Dalmau J, Graus F, Rosenblum MK et al. Anti-Hu-associated paraneoplastic encephalomyelitis/sensory neuronopathy. A clinical study of 71 patients. Medicine 1992; 71: 59-72

${ }^{6}$ Graus F, Dalmou J, Rene R et al. Anti-Hu-antibodies in patients with small cell lung cancer: association with complete response to therapy and improved survival. J Clin Oncol 1997; 15: 2866-2872

${ }^{7}$ Graus F, Keime-Guibert F, Rene R et al. Anti-Hu-associated paraneoplastic encephalomyelitis: analysis of 200 patients. Brain 2001; 124: $1138-1148$

${ }^{8}$ Dorresteijn LD, Kappelle AC, Renier WO et al. Anti-amphiphysin associated limbic encephalitis: a paraneoplastic presentation of small-cell lung carcinoma. J Neurol 2002; 249: 1307-1308 